\title{
Determinants of white-coat syndrome assessed by ambulatory blood pressure or self-measured home blood pressure

\author{
Elly Den Hond ${ }^{\mathrm{a}}$, Hilde Celis ${ }^{\mathrm{a}}$, Guy Vandenhoven ${ }^{\mathrm{b}}$, Eoin O'Brien ${ }^{\mathrm{c}}$ and Jan A. \\ Staessen ${ }^{\text {a }}$ for the THOP investigators
}

Background Gender, age, smoking, race, and body mass index have been reported to determine the ambulatory white-coat effect (WCE) and white-coat hypertension (WCH).

Methods Baseline conventional, day-time ambulatory and self-measured home blood pressure measurements from the THOP trial were used to study the effect of gender, age, body mass index, smoking habits and treatment status on the white-coat syndrome as assessed by ambulatory monitoring or self-measurement.

Results The mean systolic/diastolic WCE was 9.1/ $6.7 \mathrm{mmHg}$ if based on ambulatory blood pressure and $12.2 / 8.7 \mathrm{mmHg}$ if based on self-measured blood pressure. The ambulatory WCE was significantly higher in women, in older subjects $(65+)$, in obese subjects, in non-smokers and in patients on antihypertensive drug treatment. The self-measured WCE was significantly higher in women and in non-smokers. Ambulatory WCH was present in $6.6 \%$ of the untreated patients and $14.2 \%$ had self-measured $\mathrm{WCH}$. The proportion of ambulatory $\mathrm{WCH}$ was significantly higher in obese subjects; the proportion of self-measured $\mathrm{WCH}$

\section{Introduction}

In a previous study, we demonstrated that there is a slight but significant difference between the white-coat syndrome assessed by ambulatory monitoring and that estimated from self-measurement of blood pressure at home [1]. In this previous report, we analysed baseline data from the THOP (Treatment of Hypertension According to Home or Office Blood Pressure) trial-a study that was designed to test the hypothesis that antihypertensive drug treatment guided by the selfmeasured blood pressure may be more beneficial to the patient than treatment based on conventional blood pressure readings by the doctor [2]. The THOP trial is still ongoing, but validated baseline data on conventional, ambulatory, and self-measured home blood pressure are already available. The aim of the present analysis was to study gender, age, body mass index, smoking and treatment status as determinants of the white-coat syndrome as assessed by ambulatory monitoring or selfmeasurement. did not differ by gender, age, body mass index, or smoking habits.

Conclusions The ambulatory white-coat syndrome was determined by gender, age, body mass index, smoking habits, and treatment status. The self-measured white-coat syndrome was greater than the ambulatory white-coat syndrome but depended less on the determinants under study. Blood Press Monit 8:37-40 (c) 2003 Lippincott Williams \& Wilkins.

Blood Pressure Monitoring 2003, 8:37-40

Keywords: self-measured home blood pressure, ambulatory blood pressure, white-coat effect, white-coat hypertension

aStudy Coordinating Centre, Hypertension and Cardiovascular Rehabilitation Unit, Katholieke Universiteit Leuven, Leuven, Belgium, ${ }^{b}$ AstraZeneca NV/SA, Brussels, Belgium and ${ }^{\mathrm{c} B l o o d}$ Pressure Unit and ADAPT Centre, Beaumont Hospital, Dublin 9, Ireland.

Correspondence and requests for reprints to Elly Den Hond, $\mathrm{PhD}$, Hypertensie en Cardiovasculaire Revalidatie, Onderwijs en Navorsing, Herestraat 49, B-3000 Leuven, Belgium.

Tel: + 321634 5764; fax: + 321634 5763;

e-mail: elly.denhond@med.kuleuven.ac.be

Received 19 November 2002 Accepted 02 December 2002

\section{Methods}

\section{Study group}

The protocol of the multi-centre THOP trial was described in detail elsewhere [2]. The present study includes 474 patients of whom 366 were randomized but who all had their conventional, ambulatory and home blood pressure measured during the run-in period of the THOP trial. Previously treated patients continued their therapy during the run-in observation period.

\section{Blood pressure measurements}

The doctor measured conventional blood pressure with a standard mercury sphygmomanometer during two office visits scheduled at an interval of 2 to 3 weeks. Each time three blood pressure readings were obtained after the patient had rested for $5 \mathrm{~min}$ in the sitting position. The last two measurements of the two visits were averaged for analysis. The ambulatory blood pressure was recorded with oscillometric SpaceLabs 90207 monitors (SpaceLabs Inc., Redmond, Washington, USA), which 
were programmed to obtain blood pressure readings at 15min intervals from $0800 \mathrm{~h}$ to $2200 \mathrm{~h}$ and at $30-\mathrm{min}$ intervals for the remainder of the day. Daytime ambulatory blood pressure was calculated as the time-weighted mean of the readings obtained from $1000 \mathrm{~h}$ to $2000 \mathrm{~h}$. For the self-measurement of blood pressure at home, the patients used Omron HEM-705CP (Omron Corp., Tokyo, Japan) digital blood pressure monitors. This automated oscillometric device measures brachial artery pressure. It has successfully passed validation according to the protocol of the British Hypertension Society [3]. The patients recorded their blood pressure on seven consecutive days in the morning between $0600 \mathrm{~h}$ and $1000 \mathrm{~h}$ and in the evening between $1800 \mathrm{~h}$ and $2200 \mathrm{~h}$ with each session consisting of three readings in the sitting position. For all types of measurement, the same cuff size was used. Standard cuffs had a $24 \times 14 \mathrm{~cm}$ inflatable bladder. If arm circumference exceeded $31 \mathrm{~cm}$, larger cuffs with a bladder size of $32 \times 15 \mathrm{~cm}$ were used.

The ambulatory white-coat effect (WCE) was defined as the difference between the conventional and the daytime ambulatory blood pressure. The self-measured WCE was the difference between the conventional and the selfmeasured blood pressure. White-coat hypertension (WCH) was only diagnosed in untreated patients. The thresholds indicating hypertension were $\geq 140$ / $\geq 90 \mathrm{mmHg}$ for the conventional blood pressure and $\geq 135 / \geq 85 \mathrm{mmHg}$ for the daytime ambulatory pressure as well as for the self-measured home blood pressure [4]. Subjects with hypertension based on automated blood pressure measurement but no hypertension according to conventional measurements were classified as having 'masked WCH'.

\section{Statistical analysis}

Database management and statistical analysis were performed with SAS software (SAS Institute, Cary, NC, USA), version 8.1. Gender, age, Body mass index, smoking habits, and treatment status were considered as independent determinants of the WCE or WCH. In univariate analyses, means and proportions were compared by one-way analysis of variance or Fisher's exact test, respectively. In multivariate analyses, we used linear and logistic regression. Multiple analyses with ambulatory blood pressure measurements as dependent variables were adjusted for self-measured home blood pressure. Multiple analyses with self-measured blood pressure measurements as dependent variables were adjusted for daytime ambulatory blood pressure.

\section{Results}

\section{Patient characteristics}

The 474 patients included $252(53.2 \%)$ women, 98 (20.7\%) current smokers, 155 (32.7\%) regular alcohol consumers and $186(39.2 \%)$ patients on antihypertensive drugs. Mean age was 52.8 (SD 11.8) years. Body mass index averaged $28.1(4.7) \mathrm{kg} / \mathrm{m}^{2}$. Mean (SD) systolic/ diastolic blood pressure was $156.5(18.2) / 99.4(7.6) \mathrm{mmHg}$ for conventional measurements, $147.4(15.3) / 92.8$ (10.4) $\mathrm{mmHg}$ for the daytime ambulatory pressure and $144.4(17.5) / 90.8$ (9.7) $\mathrm{mmHg}$ for the self-measured home pressure.

Table 1 Systolic and diastolic white-coat effects (WCE) based on daytime ambulatory measurements or based on self-measured home measurement for different classes of gender, age, body mass index (BMI), smoking or treatment status

\begin{tabular}{|c|c|c|c|c|c|}
\hline & \multirow[t]{2}{*}{$n$} & \multicolumn{2}{|c|}{ Ambulatory WCE $(\mathrm{mmHg})$} & \multicolumn{2}{|c|}{ Self-measured WCE $(\mathrm{mmHg})$} \\
\hline & & Systolic & Diastolic & Systolic & Diastolic \\
\hline \multicolumn{6}{|l|}{ By gender } \\
\hline Men & 222 & $7.0(4.9-9.0)$ & $5.0(3.9-6.2)$ & $8.7(6.7-10.7)$ & $7.7(6.7-8.8)$ \\
\hline Women & 252 & $11.0(9.1-12.9)$ & $8.1(6.8-9.3)$ & $15.2(13.3-17.1)$ & $9.5(8.4-10.6)$ \\
\hline$P$ & & 0.004 & 0.005 & $<0.0001$ & 0.03 \\
\hline \multicolumn{6}{|l|}{ By age } \\
\hline Age $<45$ years & 123 & $3.8(1.5-6.1)^{\mathrm{a}}$ & $4.9(3.3-6.4)^{\mathrm{a}}$ & $11.0(8.7-13.3)$ & $9.0(7.8-10.2)$ \\
\hline Age: $45-54.9$ years & 150 & $7.0(4.7-9.3)^{\mathrm{a}, \mathrm{b}}$ & $5.3(3.7-6.8)^{\mathrm{a}}$ & $11.2(8.9-13.5)$ & $7.7(6.3-9.0)$ \\
\hline Age: $55-64.9$ years & 123 & $11.0(8.3-13.8)^{\mathrm{b}}$ & $7.8(6.0-9.5)^{a, b}$ & $12.4(9.3-15.5)$ & $8.4(6.5-10.3)$ \\
\hline Age $\geq 65$ years & 78 & $18.8(15.0-22.6)^{\mathrm{c}}$ & $10.4(8.3-11.4)^{b}$ & $15.5(11.6-19.3)$ & $10.5(8.6-12.3)$ \\
\hline$P$ & & 0.003 & $<0.001$ & 0.18 & 0.13 \\
\hline \multicolumn{6}{|l|}{ By Body mass index* } \\
\hline Normal weight & 120 & $6.7(4.4-9.1)^{\mathrm{a}}$ & $4.0(1.4-5.6)^{a}$ & $10.0(7.5-12.5)$ & $7.1(5.7-8.6)$ \\
\hline Overweight & 213 & $8.1(5.9-10.3)^{\mathrm{a}}$ & $6.0(4.8-7.3)^{\mathrm{a}}$ & $11.9(9.8-13.9)$ & $9.0(7.7-10.1)$ \\
\hline Obesity & 141 & $12.8(10.2-15.4)^{\mathrm{b}}$ & $9.8(8.2-11.4)^{\mathrm{b}}$ & $14.4(12.2-16.6)$ & $9.5(8.0-11.0)$ \\
\hline$P$ & & 0.003 & $<0.0001$ & 0.06 & 0.07 \\
\hline \multicolumn{6}{|l|}{ By smoking habits } \\
\hline Non-smokers & 376 & $10.0(8.4-11.7)$ & $7.5(6.5-8.5)$ & $12.6(11.0-14.3)$ & $9.2(8.3-10.1)$ \\
\hline Smokers & 98 & $5.7(3.3-8.1)$ & $3.3(1.8-4.9)$ & $10.4(7.9-12.9)$ & $6.7(5.1-8.4)$ \\
\hline$P$ & & 0.004 & $<0.0001$ & 0.19 & 0.01 \\
\hline \multicolumn{6}{|l|}{ By treatment status } \\
\hline No $\mathrm{AH}$ treatment & 288 & $6.5(4.9-8.2)$ & $5.1(4.0-6.1)$ & $11.3(9.6-13.0)$ & $8.4(7.4-9.3)$ \\
\hline $\mathrm{AH}$ treatment & 186 & $13.2(10.8-15.6)$ & $9.1(7.7-10.5)$ & $13.5(11.2-15.9)$ & $9.1(7.8-10.5)$ \\
\hline$P$ & & $<0.0001$ & $<0.0001$ & 0.13 & 0.37 \\
\hline
\end{tabular}

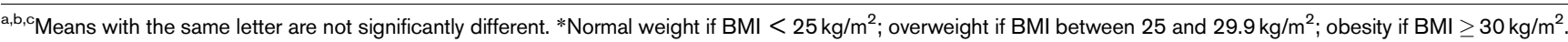


Fig. 1

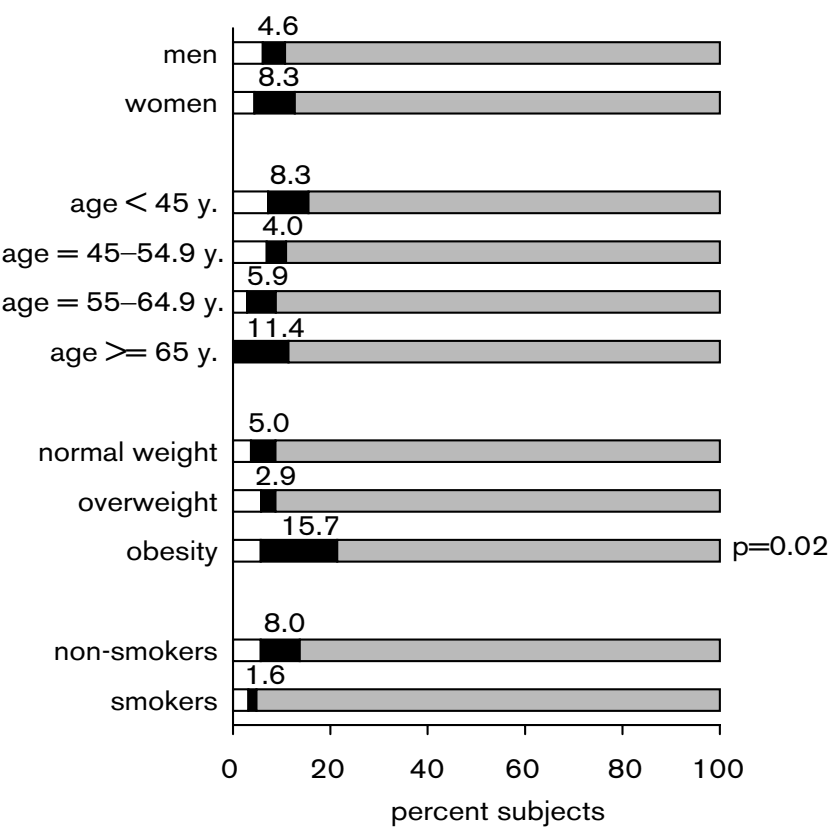

$\square$ no hypertension / masked white-coat hypertension white-coat hypertension $\square$ sustained hypertension

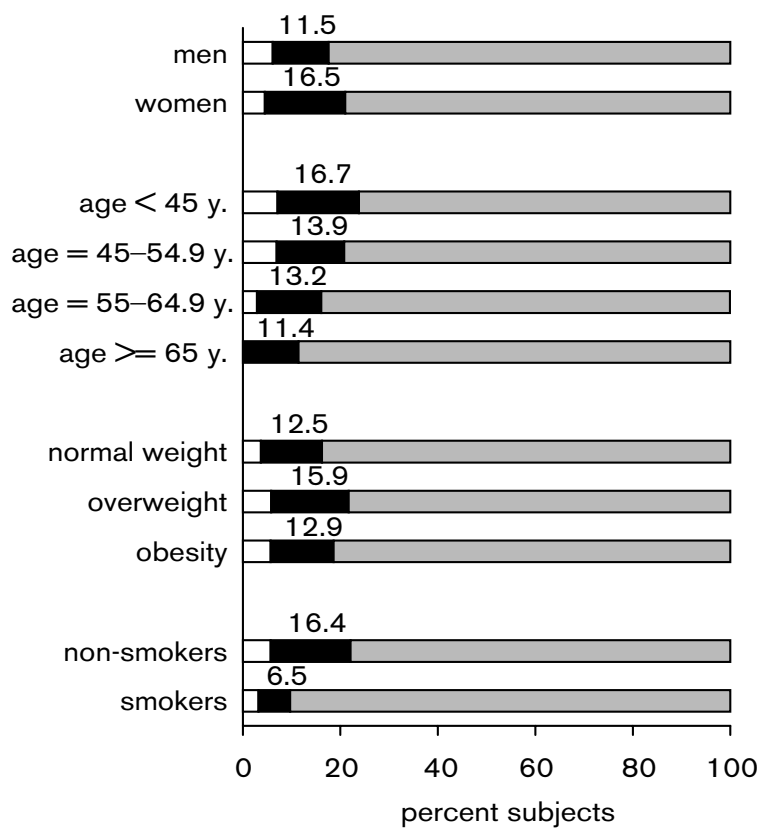

$\square$ no hypertension / masked white-coat hypertension white-coat hypertension $\square$ sustained hypertension

Percentage of subjects with 'sustained hypertension', 'white-coat hypertension' (WCH) and 'no hypertension or masked WCH' for different classes of sex, age, body mass index (BMI) or smoking habits based on ambulatory blood pressure (top panel) or self-measured home blood pressure (bottom panel). Normal weight if BMI $<25 \mathrm{~kg} / \mathrm{m}^{2}$; overweight if BMI between 25 and $29.9 \mathrm{~kg} / \mathrm{m}^{2}$; obesity if BMI $\geq$ $30 \mathrm{~kg} / \mathrm{m}^{2}$.

\section{White-coat syndrome based on ambulatory blood pressure}

Based on conventional and ambulatory daytime blood pressure measurements, the mean WCE was 9.1. (SD 15.5; range: -28.8 to 71.6$) \mathrm{mmHg}$ systolic and 6.7 (SD 9.6; range: -16.9 to 42.4$) \mathrm{mmHg}$ diastolic. In univariate analyses (Table 1), the systolic and diastolic ambulatory WCE were significantly greater in women compared with men, in non-smokers compared with smokers, and in treated compared with untreated patients. In addition, the ambulatory WCE increased with advancing age and higher body mass index. Multiple regression analyses confirmed that gender $(P<0.03)$, age $(P<0.002)$, body mass index $(P<0.04)$ and treatment status $(P<0.001)$ but not smoking $(P>0.15)$ behaved as significant and independent determinants of the systolic and diastolic ambulatory WCE.

Cross-classification of 288 untreated patients according to their conventional and ambulatory blood pressure showed sustained hypertension in $254(88.2 \%)$ patients, WCH in $19(6.6 \%)$ patients, and masked WCH in $10(3.8 \%)$ patients, whereas five $(1.7 \%)$ patients were normotensive on both measurements. In univariate analyses, the proportion of WCH patients was significantly greater in obese patients than in overweight and normal-weight subjects (Fig. 1). This conclusion was confirmed by multiple regression analysis. The relative risk of ambulatory WCH was significantly associated with body mass index $(1.15 ; 95 \%$ confidence interval, 1.02-1.30; $P=0.03$ ) but was not influenced by gender, age, or smoking habits.

\section{White-coat syndrome based on self-measured blood pressure}

Based on conventional and self-measured blood pressure measurements, the mean WCE was 12.2. (SD 15.4; range: -33.8 to 64.1$) \mathrm{mmHg}$ systolic, and 8.7 (SD 8.7; range: -33.0 to 41.9$) \mathrm{mmHg}$ diastolic. In univariate analyses of systolic pressure (Table 1), the self-measured WCE were significantly greater in women than in men, and showed a slight increase with higher body mass index $(P=0.06)$ and advancing age $(P=0.18)$. Multiple regression analysis of systolic pressure confirmed that the self-measured WCE was significantly and independently correlated with gender $(P<0.0001)$, body mass index $(P=0.04)$, and age $(P=0.02)$. In univariate analyses of the diastolic pressure (Table 1 ), the selfmeasured WCE was significantly greater in women than in men, and in non-smokers compared to smokers. Multivariate analysis, however, did not reveal significant correlates of the self-measured diastolic WCE.

Cross-classification of 288 untreated patients according to their conventional and self-measured blood pressure showed sustained hypertension in $232(80.6 \%)$ patients, 
WCH in $41(14.2 \%)$ patients, and masked WCH in seven (2.4\%) patients, whereas eight (2.8\%) patients were normotensive on both measurements. In univariate comparisons of proportions (Fig. 1) and multiple logistic regression analysis, self-measured WCH was not significantly related to any of the co-variables under study.

\section{Discussion}

In this study, we found that the ambulatory WCE was significantly higher in women, in older subjects $(65+)$, in obese subjects, in non-smokers and in patients on antihypertensive drug treatment. The self-measured WCE was significantly higher in women and in nonsmokers. The proportion of ambulatory WCH was significantly higher in obese subjects; the proportion of self-measured WCH did not differ by gender, age, body mass index, or smoking habits.

The strength of this study mainly lies in the fact that it considers a large database of standardized measurements with conventional, ambulatory, and self-measured home blood pressure available in all 474 patients.

The effects of gender, body mass index, and smoking on the ambulatory white-coat syndrome that were found in this study, are in agreement with previous reports. A greater WCE and/or a higher frequency of WCH were reported in women [5-7], in subjects with a high body mass index [6] and in non-smokers [7,8]. The effect of age is controversial: WCH has been associated with older [5,9] as well as with younger [10] age. This probably depends on the age range and the age classes that are used in the analysis.
Much less information is available on the determinants of the self-measured white-coat syndrome. According to the present results, self-measured WCE and the proportion of WCH were only to a small extent determined by gender, age, body mass index, smoking, and treatment. Whether this has any clinical implication needs further study.

\section{References}

1 Staessen JA, Celis H, Den Hond E, Giot C, Leeman M, O'Brien E, et al. Comparison of conventional and automated blood pressure measurements: interim analysis of the THOP trial. Blood Press Monit 2002; 7:61-62.

2 Celis H, Staessen JA, Buntinx F, Fagard R, Leeman M, Thijs L, Van Hedent T. Antihypertensive treatment based on home or office blood pressure measurement: protocol of the randomized controlled THOP trial. Blood Press Monit 1998; 3 (Suppl 1):S29-S35.

3 O'Brien E, Mee F, Atkins N, Thomas M. Evaluation of three devices for selfmeasurement of blood pressure according to the revised British Hypertension Society Protocol: the Omron HEM-705 CP, Philips HP5332 and Nissei DS-175. Blood Press Monit 1996; 1:55-62.

4 The Joint National Committee on Prevention Detection Evaluation and Treatment of High Blood Pressure. The Sixth report of the Joint National Committee on Prevention, Detection, Evaluation, and Treatment of High Blood Pressure. Arch Intern Med 1997; 157:2413-2446.

5 Chrysant SG. Treatment of white coat hypertension. Curr Hypertens Rep 2000; 2:412-417.

6 Gualdiero P, Niebauer J, Addison C, Clark SJ, Coats AJ. Clinical features, anthropometric characteristics, and racial influences on the 'white-coat effect' in a single-centre cohort of 1553 consecutive subjects undergoing routine ambulatory blood pressure monitoring. Blood Press Monit 2000; 5:53-57.

7 Verdecchia P, Palatini P, Schillaci G, Mormino P, Porcellati C, Pessina AC. Independent predictors of isolated clinic ('white-coat') hypertension. J Hypertens 2001; 19:1015-1020.

8 Mikkelsen KL, Wiinberg N, Hoegholm A, Christensen HR, Bang LE, Nielsen $\mathrm{PE}$, et al. Smoking related to 24-h ambulatory blood pressure and heart rate: a study in 352 normotensive Danish subjects. Am J Hypertens 1997; 10:483-491.

9 Mansoor GA, McCabe EJ, White WB. Determinants of the white-coat effect in hypertensive subjects. J Hum Hypertens 1996; 10:87-92.

10 Sandvik E, Steine S. White coat hypertension in a general practice. Prevalence, cardiovascular risk factors, and clinical implications. Scand J Prim Health Care 1998; 16:222-226. 